\title{
Fire and Smoke Identification Algorithms Based on Attention Mechanism
}

\author{
T. Luo, N.F. Xiao \\ School of Computer Science and Engineering \\ South China University of Technology \\ Guangzhou Guangdong \\ China
}

\begin{abstract}
This paper presents several fire and smoke identification algorithms based on attention mechanism, the proposed algorithms add the attention mechanism into traditional algorithms for the fire and smoke identification, which can get the possible regions of fire and smoke quickly by saliency images, and highly accelerate the computing process. Firstly, the attention mechanism is used to get the saliency images. Secondly, the Otsu method is used to segment the saliency images. Finally, ROIs (Regions of Interest) are analyzed by the fire or smoke features, in order to judge whether the fire and smoke exists or not. To judge the fire, the fire color and intensity features are used. Similarly, the color and circularity features are be used to judge the smoke.
\end{abstract}

Keywords-fire; smoke; attention mechanism; otsu; region of interest

\section{INTRODUCTION}

Conventional detection algorithms [1] for the fire and smoke disasters are widely used nowadays, and researches all over the world have done a lot of work on the algorithms, but most of the algorithms make use of some confined features, such as color, circularity and intensity features and so on. In these algorithms, in order to identify the fire and smoke, they must scan the whole image from the first pixel to last one. Thus, it is a very low efficiency, and the extracting regions of the fire or smoke are also not integrated. Attention mechanism is a relatively new technique; it can focus on a specific object quickly, and give priority to it. Therefore, it is introduced to the proposed fire and smoke identification algorithms, which can reduce the scanning area, and improve the integrity of the regions of the fire or s moke.

\section{ATTENTION MECHANISM}

It is known that human's visual system gradually formed an attention mechanism to filter unrelated information in the process of evolution; the attention mechanism is able to focus on specific significant object quickly, and give priority to it [2]. The attention mechanism can be divided into two cases: bottom-up [3] and top-down, the former is based on low-level vision, driven by data; the latter is based on advanced vision, driven by mission. This paper uses the former bottom-up attention mechanism by through various low-level features of images for generating saliency map. Its [4] uses early visual features such as color, brightness and direction, establishes a general bottom-up vision attention computational model, it does the low-level feature extraction in several spatial scales, and generates three characteristics descriptions through a combination of the feature maps which corresponds to color, brightness and direction respectively, the saliency maps are linear combination of the three significant characteristics.

Based on analyzing 5 typical detection algorithms for salient regions [5], Achanta and others put forward an algorith $\mathrm{m}$ for frequency-tuned salient region detection, which is called FT algorithm. The algorithm is simple and efficient; it can generate full resolution of the saliency images, and preserve the edges of the salient objects commendably. Achanta suggests that a saliency image should include a wide range of frequency as much as possible. Let wlcbe a low frequency cut-off value and whcbe a high frequency cut-off value, therefore, wlcshould be as low as possible, and whe should be as high as possible.

The algorithm of finding the saliency map $\mathrm{S}$ for an image I with width $\mathrm{W}$ and height $\mathrm{H}$ pixels can thus be formulated as:

$$
S(x, y)=\left|I_{\mu}-I_{w_{h c}}(x, y)\right|
$$

Where I $\mu$ is an arith metic mean pixel value of the image and Iwhc is the Gaussian blurred version of the original images to eliminate the fine texture details as well as noises and coding artifacts. I $\mu$ corresponds to the "surround", and Iwhecor responds to the "center" which Itti's algorithm mentioned.

To extend Eq. (1) to use features of color and luminance, Eq.(1) is rewrited as follows:

$$
\mathrm{S}(\mathrm{x}, \mathrm{y})=\left\|\mathrm{I}_{\mu}-\mathrm{I}_{\mathrm{w}_{\mathrm{hc}}}(\mathrm{x}, \mathrm{y})\right\|
$$

where color space here is $L \times a \times b$. I $\mu$ is a mean value of the color and luminance feature vector[Lab]T. Iwhcis the corresponding image pixel vector value in the Gaussian blurred version (using a $5 \times 5$ separable binomial kernel) of the original image, and \|\| is a normofL2.

The true usefulness of a saliency map is determined by the detail application. In this paper, the use of saliency maps is considered in the salient object segmentation, the comparisons with FT algorith $m$ are presented against the algorithm proposed by Itti(examples in fig.1). From these examples, it can be found that the FT algorithm preserves the edges of salient objects commendably, while the Itti algorithm preserves less edge information than FT algorithm. 

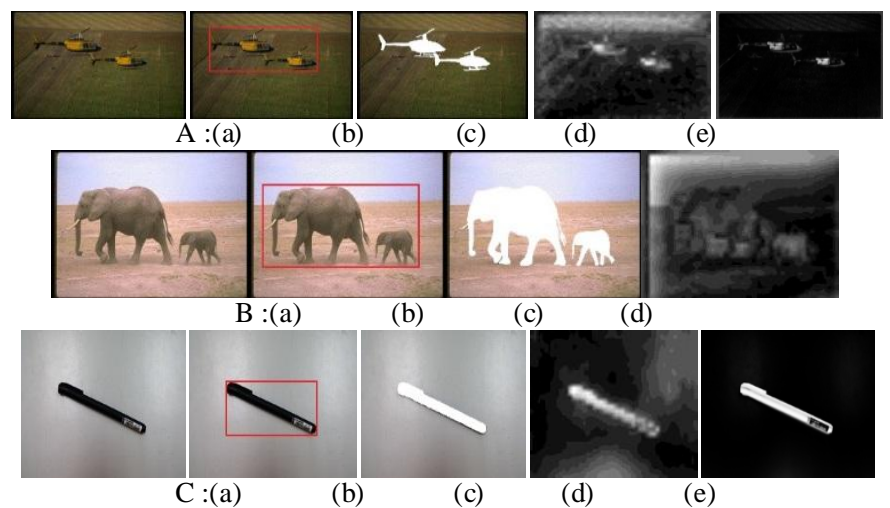

(b)

(d)

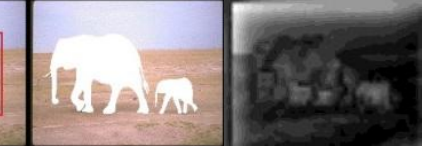

(b)

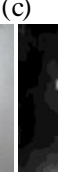

(d)

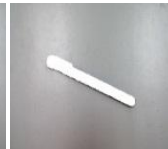

(c)

(d)

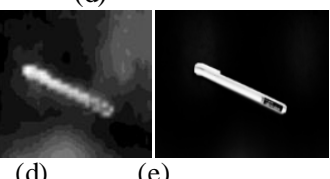

(e)

FIGURE I. (A) ISTHE ORIGINAL IMAGE. (B) IS GROUND TRUTH RECT ANGLES[6]. (C) IS GROUND TRUTH. (D)ISA SALIENCY IMAGE EXT RACTED BY ITTI ALGORITHM. (E) ISA SALIENCY IMAGE EXTRACTED BY FT ALGORITHM ADOPTED BYTHIS PAPER.

\section{FIRE COLOR FEATURES}

Due to HIS space is more suitable for identification and processing than RGB space, therefore, HIS space is used to identify the fire color features. HIS has a certain transformation relations with RGB, which can be formulated as follows:

$$
\begin{aligned}
& \mathrm{I}=\frac{\mathrm{R}+\mathrm{G}+\mathrm{B}}{3} \\
& \mathrm{~S}=1-\frac{3 \cdot \min (\mathrm{R}, \mathrm{G}, \mathrm{B})}{\mathrm{R}+\mathrm{G}+\mathrm{B}} \\
& H=\left\{\begin{array}{r}
\ddots \quad B \leq G \\
360-(, \quad B>G
\end{array}\right. \\
& \theta=\arccos \left\{\frac{[(\mathrm{R}-\mathrm{G})+(\mathrm{R}-\mathrm{B})] / 2}{\left[(\mathrm{R}-\mathrm{G})^{2}+(\mathrm{R}-\mathrm{B})(\mathrm{G}-\mathrm{B})\right]^{2}}\right\}
\end{aligned}
$$

Where, standardize $\mathrm{H}, \mathrm{S}$ and $\mathrm{Ias} \mathrm{H} \in[0,360]$, $\mathrm{S} \in[0,100], \mathrm{I} \in[0,255]$.

It can be known [7] that the value of $H, S$ and I of a fire are expressed in table 1 . According to table 1 , the fire image $f(x$, $\mathrm{y})$ can be segmented, and the image $\mathrm{g}(\mathrm{x}, \mathrm{y})$ can be gotten:

$$
\mathrm{g}(\mathrm{x}, \mathrm{y})= \begin{cases}1, & \text { if } \mathrm{f}(\mathrm{x}, \mathrm{y}) \in \mathrm{T}_{\mathrm{v}} \\ 0, & \text { else }\end{cases}
$$

where, Tvis a vector of $\mathrm{H}, \mathrm{S}$ and $\mathrm{I}$.

TABLE I. FIRE'S HUE, SATURATION AND INTENSITY.

\begin{tabular}{|l|c|c|c|}
\hline conditions & Hue & Saturation & Intensity \\
\hline bright & $0 \sim 60$ & $40 \sim 100$ & $127 \sim 255$ \\
\hline dark & $0 \sim 60$ & $20 \sim 100$ & $100 \sim 255$ \\
\hline
\end{tabular}

\section{FIRE INTENSIT Y FEATURES}

Fire region exist $\sin$ homogeneity for Intensity. Some places of the fire region are lighter than others, which forms spatiality for the fire intensity. Through extracting fire intensity features, these regions which have color features can be ruled out, but not have intensity features, therefore, firstly, calculated the average value of fire intensity:

$$
\overline{\mathrm{I}_{\text {spatial }}}=\sum_{\mathrm{j}=1}^{\mathrm{N}} \mathrm{I}\left(\mathrm{P}_{\mathrm{j}}\right) /_{\mathrm{N}}
$$

then, the change of fire intensity is defined as:

$$
I_{\text {spatial }}=\sum_{j=1}^{\mathrm{N}}\left(\mathrm{I}\left(\mathrm{P}_{\mathrm{j}}\right)-\mathrm{I}_{\text {spatial }}\right)^{2} / \mathrm{N}
$$

where, $\mathrm{I}(\mathrm{Pj})$ is the intensity value of pixel $\mathrm{Pj}$. In order to extract fire regions which have fire intensity features, a thres hold Tiis set according to the experiments. Assuming that the input image is $f(x, y)$ and the output image is $g(x, y)$, the formula can be described as follows:

$$
\mathrm{g}(\mathrm{x}, \mathrm{y})=\left\{\begin{array}{l}
0, f(x, y)<\mathrm{T}_{\mathrm{i}} \\
1, f(x, y) \geq \mathrm{T}_{\mathrm{i}}
\end{array}\right.
$$

\section{V.SMOKE COLOR FEATURES}

Similarly, Smoke has color feature in color space. In the early fire, s moke presents pewter. In RGB space, the snowks color features cannot be separated from red, green or blue components to determine. It can be determined jointly by red, green and blue components. It can be found that the three components have approximately equal values. Letabe a parameter to represent similar degree of R, G and B, and it can be defined as:

$$
\alpha=\max (|R-G|,|G-B|,|B-R|)
$$

where, the range of values for $\alpha$ is 15 to 20 after a lot of experiments.

In HIS space, the value ranges for $\mathrm{H}$ is 175 to 185 . According to the smoke color features and HIS color distance, the pixels which have the same color feature can be clustered in a class, and others in another class. This algorithm process is shown in fig. 2 .

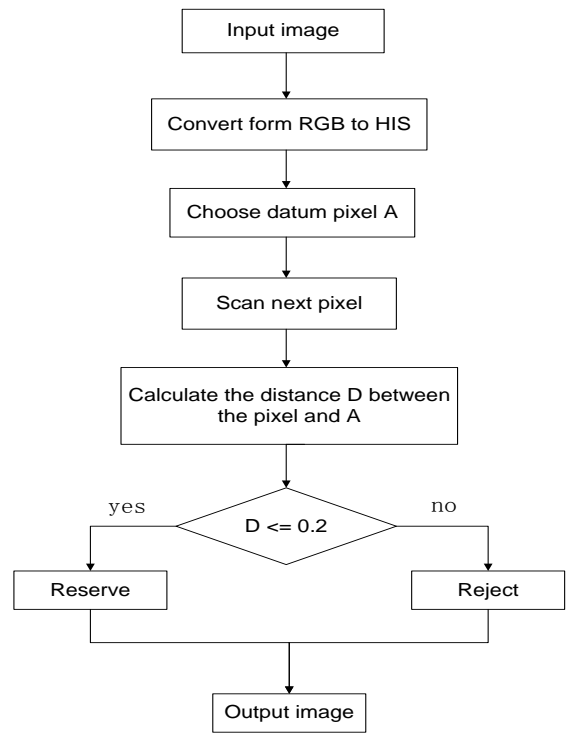

FIGURE II. FLOWCHART OF SMOKE COLOR FEATURES 
(1) Choose datum pixel: scan pixel $X$ one by one, and choose the one which meets the value ranges for $\boldsymbol{\alpha}$ and $\boldsymbol{H}$ as datum pixel $\mathrm{A}$.

(2) Calculate HIS value of next pixel: scan next pixel B, and calculate its values of $\boldsymbol{H}, \boldsymbol{I}$ and $\boldsymbol{S}$.

(3) Calculate the distance $\boldsymbol{D}$ between A and B:

$$
\begin{gathered}
\mathrm{D}=\sqrt{\mathrm{V}_{1}+\mathrm{V}_{2}+\mathrm{V}_{3}} \\
\mathrm{~V}_{1}=(\mathrm{I}-\mathrm{i})^{2} \\
\mathrm{~V}_{2}=\mathrm{S} \cdot \cosh -\mathrm{s} \cdot \cosh \cdot(\mathrm{S} \cdot \cos \mathrm{H}-\mathrm{s} \cdot \cosh ) \\
\mathrm{V}_{3}=\mathrm{S} \cdot \cos \mathrm{H}-\mathrm{s} \cdot \sinh \cdot(\mathrm{S} \cdot \sinh -\mathrm{s} \cdot \sinh )
\end{gathered}
$$

(4) Reserve the pixel which has smoke color features: when $\mathrm{D}$ is less than 0.2 , reserve the pixel, repeat steps (2)-(4).

\section{SMOKE CIRCULARITY FEATURES}

As the smoke represents irregular shape, the property can be used to exclude these objects which has smoke color features, but has not irregular shape in background. The irregular shapes of smoke will change with air flow, it is difficult to measure the shape. Therefore, the circularity is used to indicate the complexity of the shape. The circularity is defined as:

$$
\mathrm{C}=\mathrm{L}^{2} / 4 \pi \mathrm{S}
$$

where, Lis perimeter, and Sis area. When an object is a circle, its circularity value Cis 1 which is the minimal value.

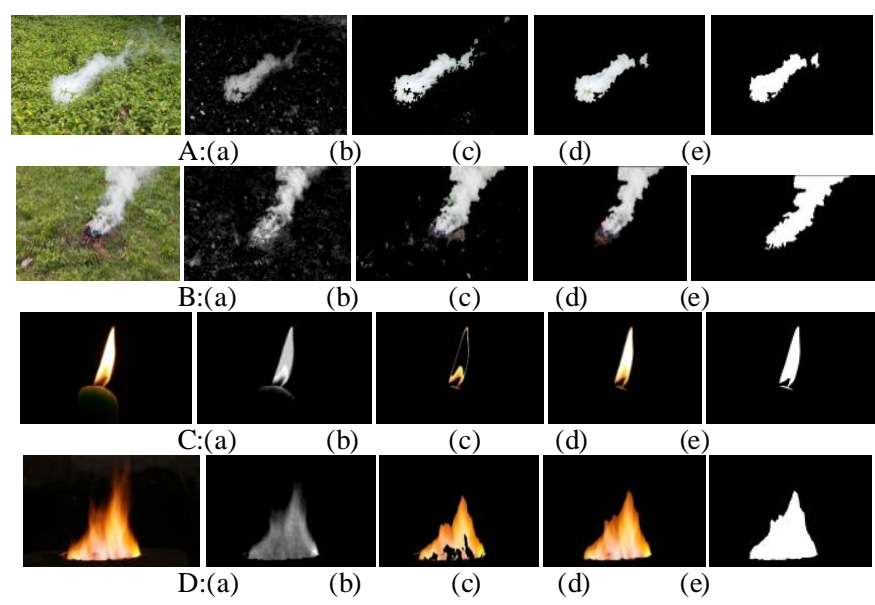

FIGURE III. (A) IS ORIGINAL IMAGE. (B) IS SALIENCY IMAGE EXT RACTED BY FT ALGORITHM ADOPTED BYTHIS PAPER. (C) ISA SMOKE OR FIRE IMAGE IDENT IFIED BYT RADITIONAL ALGORIT HM. (D) ISA SMOKE OR FIRE IMAGE IDENTIFIED BY THE PROPOSED METHOD. (E) IS (D)'S BINARY IMAGE.

\section{COMPARISONS}

The true usefulness of a saliency map is determined by its application. The use of saliency maps in this paper is considered in the fire and smoke identification. To segment the fire and smoke regions, we need to extract saliency objects of original image firstly, and then judge these object whether meet the features of fire or smoke. The comparisons with the proposed algorithm in this paper are presented against the traditional ones (examples in fig.3). In the first experiment, a smoke image is used as input image. In the second experiment, a fire image is used as input image.

It is easy to know from the experimental results that the proposed algorithm is better than the traditional algorithms. The traditional algorithms base on pixel, while the proposed algorithm bases on saliency region. Therefore, the fire or smoke images in traditional algorithms are incomplete and noisy, while the fire or smoke images in the proposed algorithm are integrated and unadulterated.

\section{CONCLUSIONS}

This research develops a fire and smoke algorithm based on the attention mechanism to provide an early alarm for the fire accidents. The attention mechanism is used into the algorithm to improve the efficiency and reliability of the fire and smoke detection. The experimental results show that the proposed algorithm can provide a reliable and cost-effective solution for the fire and smoke detection, and it may be more attractive than the traditional algorithms of the fire and smoke detection.

\section{ACKNOWLEDGEMENT S}

This research in this paper is supported by the National Natural Science Foundation (Project No.61171141) and the province ministry cooperation projects of special funds, Guangdong Province (Project No. 2012B091100448), the authors express sincere thanks for the foundation.

\section{REFERENCES}

[1] Maruta, H., Kato, Y., Nakamura, A., \& Kurokawa, F. (2009, July). Smoke detection in open areas using its texture features and time series properties. In Industrial Electronics, 2009. ISIE 2009. IEEE International Symposium on (pp. 1904-1908). IEEE.

[2] Hurmuzlu, Y., Génot, F., \&Brogliato, B. (2004). Modeling, stability and control of biped robots - a general framework. Automatica, 40(10), 1647-1664.

[3] Gao, D., \&Vasconcelos, N. (2007, October). Bottom-up saliency is a discriminant process. In Computer Vision, 2007. ICCV 2007. IEEE 11th International Conference on (pp. 1-6). IEEE.

[4] Itti, L., Koch, C., \&Niebur, E. (1998). A model of saliency-based visual attention for rapid scene analysis. IEEE T ransactions on pattern analysis and machine intelligence, 20(11), 1254-1259.

[5] Achanta, R., Hemami, S., Estrada, F., \&Susstrunk, S. (2009, June). Frequency-tuned salient region detection. In Computer Vision and Pattern Recognition, 2009. CVPR 2009. IEEE Conference on (pp. 15971604). IEEE.

[6] Wang, Z., \& Li, B. (2008, March). A two-stage approach to saliency detection in images. In Acoustics, Speech and Signal Processing, 2008. ICASSP 2008. IEEE International Conference on (pp. 965-968). IEEE.

[7] Horng, W. B., Peng, J. W., \& Chen, C. Y. (2005, March). A new imagebased real-time flame detection method using color analysis. In Networking, Sensing and Control, 2005. Proceedings. 2005 IEEE (pp. 100-105). IEEE. 